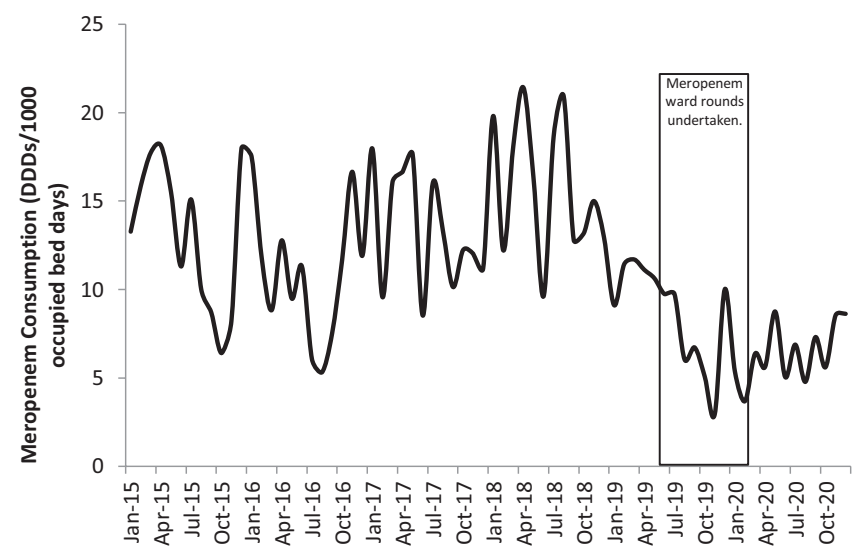

Fig. 1. Southern Health and Social Care Trust (SHSCT) meropenem consumption between January 2015 and December 2020; vertical lines represent the intervention period (June 2019-February 2020).

rounds were suspended. Previous studies have demonstrated the long-term benefits of the "handshake stewardship" approach in pediatric healthcare settings. ${ }^{9,10}$ MacBrayne et al ${ }^{9}$ observed decreases on total antibiotic use for up to 5 years after implementing an intervention consisting of ID physician or antimicrobial pharmacist review of patients regarding antibiotic consumption. ${ }^{9}$ The in-person approach used in the present study allowed a full patient assessment to be performed followed by face-to-face conversations with the clinical team in a nonconfrontational manner.

This study demonstrates the importance of an in-person review by the AST team, which has enabled rationalizing of antibiotic therapy, resulting in significant reductions in meropenem prescribing. The AST-led meropenem reviews also produced added benefits through the education of clinical teams following faceto-face case discussions, which have the potential to have long-lasting effects beyond the duration of the intervention.

Acknowledgments. This project was considered a service evaluation, and ethical approval was not required. This study was registered with the Health Trust audit department.
Financial support. This study was carried out as part of our routine work.

Conflicts of interest. All authors report no conflicts of interest relevant to this article.

\section{References}

1. NI Department of Health, Department of Agriculture, Environment and Rural Affairs, Foods Standards Agency. Changing the culture 2019-2024: one health. Tackling antimicrobial resistance in Northern Ireland: a fiveyear action plan. Northern Ireland Department of Health website. https://www.health-ni.gov.uk/sites/default/files/publications/health/onehealth-antimicrobial.pdf. Published 2019. Accessed April 9, 2020.

2. Davey P, Brown E, Charani E, et al. Interventions to improve antibiotic prescribing practices for hospital inpatients. Cochrane Database Syst Rev 2013;(4):CD003543.

3. Moghnieh R, Awad L, Abdallah D, et al. Effect of a "handshake" stewardship program versus a formulary restriction policy on high-end antibiotic use, expenditure, antibiotic resistance, and patient outcome. J Chemother 2020; 32:368-384.

4. Jirjees FJ, Al-Obaidi HJ, Sartaj M, et al. Antibiotic use and resistance in hospitals: time-series analysis strategy for determining and prioritising interventions. Hosp Pharm Eur 2020;95:13-19.

5. Garcia-Rodriguez JF, Bardan-Garcia B, Pena-Rodriguez MF, Alveraz-Diaz H, Marino Callejo A. Meropenem antimicrobial stewardship program: clinical, economic, and antibiotic resistance impact. Eur J Clin Microbiol Infect Dis 2019;38:161-170.

6. Seah VXF, Ong RYL, Lim ASY, Chong CY, Tan NWH, Thoon KC. Impact of a carbapenem antimicrobial stewardship program on patient outcomes. Antimicrob Agents Chemother 2017;61:e00736-17.

7. Suzuki A, Maeda M, Yokoe T, Hashiguchi M, Togashi M, Ishino K. Impact of the multidisciplinary antimicrobial stewardship team intervention focusing on carbapenem de-escalation: a single-centre and interrupted time series analysis. Int J Clin Pract 2020;75:e13693.

8. Horikoshi Y, Suwa J, Higuchi H, et al. Sustained pediatric antimicrobial stewardship program with consultation to infectious diseases reduced carbapenem resistance and infection-related mortality. Int $J$ Infect Dis 2017;64:69-73.

9. MacBrayne CE, Williams MC, Levek C, et al. Sustainability of handshake stewardship: extending a hand is effective years later. Clin Infect Dis 2020; 70:2325-2332.

10. Hurst A, Child J, Parker S. Intervention and acceptance rates support handshake stewardship. J Pediatric Infect Dis Soc 2019;8:162-165.

\title{
Strategy to limit multidrug-resistant Acinetobacter baumannii transmission in a cohort coronavirus disease 2019 (COVID-19) critical care unit
}

\author{
Anucha Apisarnthanarak MD ${ }^{1}$ (1) and David J. Weber MD, MPH \\ ${ }^{1}$ Division of Infectious Diseases, Thammasat University Hospital, Pathum Thani, Thailand and ${ }^{2}$ Gillings School of Global Public Health, Chapel Hill, \\ North Carolina, United States
}

\footnotetext{
Author for correspondence: Anucha Apisarnthanarak, Email: anapisarn@yahoo.com Cite this article: Apisarnthanarak A and Weber DJ. (2022). Strategy to limit multidrugresistant Acinetobacter baumannii transmission in a cohort coronavirus disease 2019 (COVID-19) critical care unit. Infection Control \& Hospital Epidemiology, 43: 1517-1518, https://doi.org/10.1017/ice.2021.289
}

To the Editor-Coinfection with multidrug-resistant organisms (MDROs) among coronavirus disease 2019 (COVID-19) patients is common in critical care patients with a prolonged length of stay in critical care units, likely due to the coadministration of high-dose steroids and the prolonged duration of mechanical ventilation. ${ }^{1}$ The control of MDROs among COVID-19 patients 
is also difficult, given the requirement for airborne plus contact isolation among these patients and the difficulty in wearing and changing personal protective equipment (PPE) in a critical care unit. ${ }^{2}$ The situation is much more challenging in middle- and lower-income countries where cohort areas in airborne-isolation critical care units are often designed, instead of single airborneisolation rooms in the critical care unit. We report the experience of controlling an MDR Acinetobacter baumannii outbreak in a COVID-19 critical care unit that featured airborne isolation cohorting areas together with limited standard single airborne isolation rooms in Thailand.

On March 1, 2021, at Thammasat University Hospital (Pratum Thani, Thailand), the first case of MDR A. baumannii was detected in a COVID-19 critical care unit, followed by 1 additional patient who was located next to the index patient 2 days later in the same cohorting area. In this 10-bed critical care unit, there were 2 sections of 4 -bed airborne isolation cohort areas and 2 singleroom beds which were airborne isolation rooms. The nurseto-patient ratio in this unit was 2.5 to 1 . After the detection of the first case, a root-cause analysis revealed the possibility of cross transmission because healthcare personal (HCP) are unable to change PPE between caring for patients in the cohort area as well as the possibility of an unrecognized case of MDR A. buamannii referred from another hospital. A policy to prevent transmission was initiated that included isolation of MDR A. baumannii patients in single-bed isolation rooms, assigning specific nurses to care for cases with MDR A. baumannii, changing gloves between cases, putting an extra sheet cover on the provider between care for MDR A. baumannii cases and daily environmental disinfection in cohort and single beds with a quaternary ammonium compound. Feedback regarding compliance with infection prevention practices by HCP was given daily. After 2 weeks, 4 additional MDR A. baumannii cases occurred in the cohort area (the incidence rate, 16.9 cases per 1,000 patient days), despite full compliance with policies. A subsequent root-cause analysis suggested the possibility of widely disseminated environmental contamination with MDR A. baumannii together with the possibility of cross transmission of MDR A. baumannii by HCP unable to change gowns between patients in the cohort area.

Additional interventions at this stage included unit closure for hydrogen peroxide vapor disinfection, development of risk stratification criteria for housing high-risk patients with MDR A. baumannii in the 2 isolation rooms, development of an antibiotic stewardship program to limit broad-spectrum antibiotics and to de-escalate broad-spectrum antibiotics among COVID-19 patients, and development of a policy to discontinue isolation for COVID-19 patients. $^{3}$ Continuous monitoring and feedback of MDR A. baumannii incidence and infection prevention compliance with such policies among HCP was performed daily. During the subsequent 8 weeks, 2 additional cases of MDR A. baumannii were detected. A significant reduction in MDR A. baumannii incidence occurred compared to the period before the intervention: 16.9 cases per 1,000 patient days versus 3.6 cases per 1,000 patient days $(P<$ $.001)$. Infection prevention compliance monitoring among HCP indicated full compliance with all components of infection prevention.

It is well recognized that MDR A. baumannii are selected by use of broad-spectrum antibiotics and often have an environmental reservoir that can facilitate rapid spread in critical care units if appropriate interventions are not introduced. ${ }^{4,5}$ Also, the constant use of gloves and gowns during a SARS outbreak led to an increase in transmission of MDROs, particularly methicillin-resistant Staphylococcus aureus. ${ }^{6}$ Outbreaks of MDR A. baumannii can be more difficult to control in middle- and lower-income countries where infrastructure may not be adequate (eg, suboptimal design of negative pressure airborne isolation units, inadequate nurseto-patient ratio). Such conditions require a practical strategy to control MDROs in the resource limited settings. Our experience suggests that policies that using an additional sheet to protect contamination of HCP gowns, frequent changes of gloves, assignment of specific nurses to care for MDR A. baumannii cases as well as basic environmental disinfection were not able to terminate an outbreak of MDR A. baumannii if the HCP could not change PPE between patients in cohort areas. Additional strategies are needed in situations that do not allow changing PPE easily between cases in the cohort areas. These strategies must feature multimodal approaches that include risk stratification for index patients that may potentially harbor MDR A. baumannii with isolation in single rooms, an antibiotic stewardship program for COVID-19 patients, and policies to discontinue COVID-19 isolations as well as a policy to perform robust terminal environmental disinfection. Such strategies, together with fully compliance with infection prevention measures, will help limit the transmission of MDR A. baumannii in COVID-19 cohort areas in critical care units. Additional studies to evaluate practical strategies to help limit transmission of MDR-pathogens in cohort-type COVID-19 critical care units are needed.

\section{Acknowledgments.}

Financial support. No financial support was provided relevant to this article.

Conflicts of interest. All authors report no conflicts of interest relevant to this article.

\section{References}

1. Karruli A, Boccia F, Gagliardi M, et al. Multidrug-resistant infections and outcome of critically ill patients with coronavirus disease 2019: a single center experience. Microb Drug Resist 2021. doi: 10.1089/mdr.2020.0489.

2. Lynch JB, Davitkov P, Anderson DJ, et al. Infectious Diseases Society of America guidelines on infection prevention for healthcare personnel caring for patients with suspected or known COVID-19. Clin Infect Dis 2020. doi: 10.1093/cid/ciaa1063.

3. Rhee C, Kanjilal S, Baker M, Klompas M. Duration of severe acute respiratory syndrome coronavirus 2 (SARS-CoV-2) infectivity: when is it safe to discontinue isolation? Clin Infect Dis 2021;72:1467-1474.

4. Ng TM, Teng CB, Lye DB, Apisarnthanarak A. A multicenter case-case control study for risk factors and outcomes of extensively drug-resistant Acinetobacter baumannii bacteremia. Infect Control Hosp Epidemiol 2014;35: 49-55.

5. Teerawattanapong N, Kengkla K, Dilokthornsakul P, Saokaew S, Apisarnthanarak A, Chaiyakunapruk N. Prevention and control of multidrugresistant gram-negative bacteria in adult intensive care units: a systematic review and network meta-analysis. Clin Infect Dis 2017;64 suppl 2:S51-S60.

6. Yap FHY, Gomersall CD, Fang KSC, et al. Increase in methicillin-resistant Staphylococcus aureus acquisition rate and change in pathogen pattern associated with outbreak of severe acute respiratory syndrome. Clin Infect Dis 2004;39:511-516. 